\title{
Revue de l'état des connaissances des effets du tritium sur la santé et l'environnement au Canada - un outil pour orienter la surveillance réglementaire
}

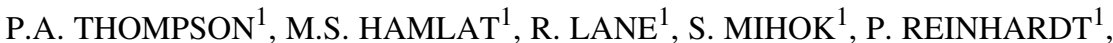
K. BUNDY ${ }^{1}$

(Manuscrit reçu le 27 mai 2011, accepté le 26 septembre 2011)

Résumé L'utilisation du tritium dans les réacteurs CANDU (Canada Deutérium Uranium), dans l'industrie, pour la production de sources et de peintures luminescentes, dans l'exploration pétrolière et gazière, dans les hôpitaux pour des tests diagnostiques, en radiothérapie et en recherche, rend le contrôle de ses rejets particulièrement important au Canada. Ces rejets sont réglementés et étroitement surveillés par la Commission canadienne de sûreté nucléaire (CCSN). Certains groupes d'intérêt et de citoyens prétendent cependant quel'incertitude scientifique sur les effets du tritium sur la santé et l'environnement est telle que la réglementation des installations rejetant ou utilisant du tritium serait inadéquate. Pour répondre à ces inquiétudes, la CCSN a demandé à son personnel d'entreprendre le projet intitulé «Études sur le tritium ». Dans le cadre de ce projet, le devenir du tritium dans l'environnement ainsi que ses effets sanitaires, ont été étudiés au moyen de mesures directes sur le terrain ainsi qu'à travers la revue de la littérature scientifique la plus récente sur le sujet. Ce projet a permis de conclure que les mesures de radioprotection concernant le tritium ainsi que les mécanismes utilisés pour le réglementer sont adéquats pour protéger la santé et la sécurité des Canadiens.

Abstract Review of the knowledge available to date on the effects of tritium exposure on health and the environment in Canada - a tool to guide regulatory compliance monitoring. The use of tritium in CANDU (Canadian Deuterium-Uranium) reactors, in industry to produce self-luminescent lights and paints, in oil and gas exploration, in hospitals for diagnostic tests and radiotherapeutics, and in research makes the control of tritium releases generated by these activities particularly important in Canada. Releases are regulated and carefully monitored by the Canadian Nuclear Safety Commission (CNSC). Some special interest and citizen groups, however, claim that the scientific uncertainty regarding the effects of tritium on health and on the environment is such that regulation of the facilities releasing or using tritium may be inadequate. In response to these concerns, the CNSC asked its staff to initiate the "Tritium Studies" project. As part of the project, the environmental fate of tritium and its health effects were studied through direct field measurements and the review of the latest scientific literature on the subject. The project made it possible to conclude that the tritium radiation protection measures and regulatory mechanisms are adequate in protecting the health and safety of Canadians.

Keywords: CANDU / tritium / environment / health effects / RBE

1 Commission canadienne de sûreté nucléaire, Direction de l'évaluation et de la protection environnementales et radiologiques, 280 rue Slater, Ottawa, Ontario, K1P 5S9, Canada. 
P.A. THOMPSON et al.

\section{Introduction}

Au Canada, le contrôle des rejets du tritium dans l'environnement revêt une importance particulière parce que les réacteurs nucléaires de type CANDU (Canada Deutérium Uranium) qui y sont utilisés, produisent d'importantes quantités de tritium. A ces rejets, s'ajoutent ceux des autres installations qui manipulent le tritium en grandes quantités, notamment les installations de récupération et de traitement du tritium, ainsi que celles qui fabriquent des sources radioactives et des peintures luminescentes. Le tritium est aussi utilisé dans les hôpitaux à des fins diagnostiques ou thérapeutiques, dans les laboratoires de recherche, comme traceur dans l'exploration pétrolière et gazière et en hydrogéologie et en hydrologie (Galeriu et al., 2005 ; Weiss et al., 1979 ; WHO, 1983).

Les rejets de tritium générés par ces activités sont réglementés et rigoureusement surveillés par la Commission canadienne de sûreté nucléaire (CCSN).

Au Canada et ailleurs, certains représentants de groupes d'intérêt ainsi que certains citoyens prétendent que l'incertitude scientifique sur les effets du tritium sur la santé et l'environnement est telle que la réglementation des installations nucléaires utilisant ou rejetant du tritium dans l'environnement ne serait pas assez sévère. Certains ont même déclaré que les femmes en âge de procréer ne devraient pas habiter en deçà d'un rayon de 10 kilomètres autour d'une centrale nucléaire (Greenpeace Report, 2007).

Plusieurs agences gouvernementales ont répondu à ces inquiétudes en mandatant une revue des connaissances et une évaluation de la pertinence de réviser la réglementation actuelle (HPA, 2007; ASN, 2010). C'est dans ce contexte, qu'en janvier 2007, le tribunal de la CCSN a demandé à son personnel d'entreprendre le projet intitulé «Études sur le tritium ». L'objectif de ce projet était de revoir l'information scientifique sur les effets du tritium sur la santé et l'environnement, disponible à ce jour, afin de s'en servir pour mieux orienter la surveillance réglementaire.

Le présent article met l'accent sur le devenir du tritium dans l'environnement ainsi que sur ses effets potentiels sur la santé. Certaines données récoltées dans le cadre du projet ainsi que les conclusions et recommandations qui en découlent sont présentées ici. Toutefois, pour obtenir une vision d'ensemble du projet, il est suggéré de consulter dans leur intégralité, sur le site Web de la $\mathrm{CCSN}^{2}$, tous les rapports qui en découlent.

2 http://www.nuclearsafety.gc.ca/fr/readingroom/tritium/tritium_studies.cfm 


\section{Méthodes}

Le premier objectif du projet était de rassembler des données sur l'état et la nature des impacts des rejets de tritium associés à toutes les installations et activités autorisées d'importance au Canada. Ces données ont par la suite été compilées et interprétées selon leur impact radiologique sur les deux groupes suivants: les travailleurs du secteur nucléaire et le public (groupe critique). Les niveaux d'activité du tritium dans l'environnement ont été compilés de façon détaillée à partir des rapports de conformité soumis à la CCSN par les titulaires de permis.

Le deuxième objectif était d'examiner les techniques de contrôle (mesures d'atténuation) des rejets par les installations de production, de manipulation ou de gestion du tritium au Canada. Toutes les grandes installations du Canada ainsi que certaines installations à l'étranger (Grande Bretagne, Suisse et Afrique du Sud) ont été visitées et évaluées dans le cadre de ce projet. Les technologies, les pratiques et le rendement en matière d'exploitation des installations canadiennes ont été évalués en fonction des meilleures pratiques internationales.

Le troisième objectif était d'étudier le devenir du tritium rejeté dans l'atmosphère en se basant sur des données de conformité environnementale fournies à la CCSN par les détenteurs de permis. Dans un premier temps, une revue et une évaluation critique de la littérature scientifique courante sur les voies de transfert du tritium dans l'environnement et sur le devenir du tritium rejeté dans l'atmosphère ont été effectuées. Ces informations techniques et scientifiques sont à la base des modèles mathématiques $(\mathrm{CSA}, 2008)^{3}$ utilisés pour prédire le comportement dans l'environnement du tritium de source industrielle. Dans un deuxième temps, l'activité du tritium dans l'air, les sols, les eaux de surface et les eaux souterraines dans le voisinage des installations nucléaires a été comparée aux prédictions du modèle mathématique choisi.

Dans le cadre du troisième objectif du projet, le personnel de la CCSN a effectué des mesures indépendantes de deux composés tritiés, soit l'eau tritiée (HTO) et le tritium lié organiquement (OBT), dans les sols, les produits maraîchers ainsi que dans d'autres aliments produits localement. Des études expérimentales sur la dynamique d'absorption du tritium dans les sols et la végétation ont aussi été menées autour de deux centrales nucléaires et de deux installations de traitement du tritium afin d'évaluer les différents types de rejets (HTO ou HT (hydrogène tritié)).

\footnotetext{
3 CSA N288.1-08: Guidelines for calculating derived release limits for radioactive material in airborne and liquid effluents for normal operation of nuclear facilities disponible à l'adresse suivante : http://www.shopcsa.ca/onlinestore/GetCatalogItemDetails.asp?mat=2419522
} 
Le quatrième objectif était d'effectuer une revue indépendante de la littérature scientifique disponible à ce jour afin d'évaluer, les risques sanitaires pour les individus exposés au tritium, les pratiques de dosimétrie du tritium employées au Canada et ailleurs, et la pertinence des limites réglementaires en place en matière de radioprotection.

Certains résultats des volets un, trois et quatre du projet sont présentés ici.

\section{Résultats}

\subsection{Activité du tritium dans l'environnement et conséquences sur les doses au public}

Les rejets de tritium dans l'air et dans l'eau pour toutes les installations canadiennes ont été compilés pour l'année 2006 (CSN, 2009) : toutes les centrales nucléaires (Pointe Lepreau au Nouveau-Brunswick, Pickering A et B en Ontario et Gentilly2 au Québec) à l'exception de la centrale de Darlington en Ontario n'ont rejeté que de l'oxide de tritium (HTO). La centrale de Darlington a, de son côté, rejeté aussi une certaine quantité de tritium gazeux (HT), ce qui représentait environ $75 \%$ de ses rejets de HTO. Les deux installations de traitement du tritium (SRB Technologies (SRBT) et Shield Source Inc. (SSI)) ont rejeté du tritium majoritairement sous forme de HT. La somme de ces rejets s'est avérée ne représenter qu'une faible fraction de la limite réglementaire de $1 \mathrm{mSv}$ par année pour le public.

L'activité du tritium dans l'air, mesurée dans le voisinage de toutes les installations, variait entre 0,4 Bq. $\mathrm{m}^{-3}$ et $35,7 \mathrm{~Bq} \cdot \mathrm{m}^{-3}$, tandis que l'activité provenant du bruit de fond régional mesurée loin des installations nucléaires était d'environ $0,1 \mathrm{~Bq} \cdot \mathrm{m}^{-3}$.

Dans le voisinage immédiat des centrales nucléaires, les concentrations de tritium dans les précipitations, les sources d'eau potable urbaines, les puits hors site, les eaux de surface et le lait étaient en général inférieures à $50 \mathrm{~Bq} . \mathrm{L}^{-1}$ tandis que le bruit de fond régional représentait entre 2 et $7 \mathrm{~Bq} \cdot \mathrm{L}^{-1}$. Quelques concentrations de tritium plus élevées ont été mesurées dans les précipitations autour de la centrale nucléaire de Pickering en Ontario (493 Bq.L ${ }^{-1}$ ) et dans les eaux de surface près de la zone d'entreposage à sec du combustible nucléaire usé de la centrale nucléaire Gentilly-2 (892 Bq.L $\left.{ }^{-1}\right)$ au Québec. L'activité du tritium dans les légumes, les fruits, l'ensilage, le miel et les animaux terrestres et aquatiques des environs était généralement inférieure à $50 \mathrm{~Bq} \cdot \mathrm{L}^{-1}$, à l'exception de la viande de cerf de Virginie, dans les environs de la centrale nucléaire de Bruce en Ontario, dans laquelle l'activité mesurée atteignait $819 \mathrm{~Bq} . \mathrm{L}^{-1}$, et des fruits cultivés aux alentours de la centrale nucléaire de Pickering en Ontario, où elle atteignait $194 \mathrm{~Bq} . \mathrm{L}^{-1}$. 
Les concentrations de tritium mesurées dans l'eau de surface, la viande, le lait, les légumes et les fruits, autour des installations de traitement du tritium et des Laboratoires d'Énergie Atomique Canada limitée (EACL) à Chalk River (LCR) en Ontario (CSN, 2009), étaient parfois plus élevées que les valeurs mesurées autour des centrales nucléaires. Dans le cas des deux installations de traitement du tritium (SRBT et SSI) rejetant principalement du tritium sous forme HT, les échantillons ont été prélevés plus près des points de rejet de tritium dans l'atmosphère que pour les centrales nucléaires, où les zones d'exclusion sont très larges, et l'activité du tritium dans les légumes et les fruits cultivés autour de ces installations atteignait des valeurs moyennes avoisinant les $3091 \mathrm{~Bq} \cdot \mathrm{L}^{-1}$.

Les concentrations de tritium dans les sources d'eau potable urbaines près des centrales nucléaires font l'objet d'une surveillance régulière et systématique. Les valeurs moyennes, en 2006, fluctuaient entre 7 et 18 Bq.L ${ }^{-1}$. Les concentrations de tritium autour des centrales nucléaires étaient du même ordre de grandeur et sont toujours demeurées inférieures à la limite de 7000 Bq.L ${ }^{-1}$ préconisée dans les Recommandations pour la qualité de l'eau potable au Canada ${ }^{4}$. Cette valeur est différente des limites adoptées dans plusieurs autres pays ; par exemple, l'Union européenne, les États-Unis, l'Australie, la Finlande et la Russie, ont respectivement adopté des limites de tritium dans l'eau potable de 100, 740, 76103 et $30000 \mathrm{~Bq} . \mathrm{L}^{-1}$. Quant à l'Organisation mondiale pour la santé (OMS) chargée de la direction et de la coordination de la santé au sein du réseau des Nations Unies, elle recommande une limite de 10000 Bq.L ${ }^{-1}$.

La valeur la plus élevée mesurée dans un puits d'eau potable en 2006 a été de $1875 \mathrm{~Bq} . \mathrm{L}^{-1}$. Il s'agissait du puits d'une résidence près d'une installation de traitement du tritium mais qui ne sert plus de source d'eau potable. En 2010, dû à un contrôle accru des rejets, la concentration de tritium dans le même puits avait diminué jusqu'à 423 Bq. $\mathrm{L}^{-1}$.

En 2006, les doses de rayonnement attribuables à l'exposition au tritium pour les individus vivant à proximité des centrales nucléaires ont varié entre 0,45 et $2,36 \mu \mathrm{Sv} \mathrm{an}^{-1}$ (Fig. 1). Les estimations des doses de tritium au public vivant à proximité des installations de traitement du tritium étaient également faibles $(0,01$ à $14,5 \mu \mathrm{Sv} . \mathrm{an}^{-1}$ ), sauf dans le cas de SSI, où la dose a été établie à $67 \mu \mathrm{Sv} . \mathrm{an}^{-1}$ en se basant sur des estimations très prudentes pour un individu du groupe critique ${ }^{5}$.

\footnotetext{
4 http://www.ccme.ca/assets/pdf/rqec_pn_1041.pdf

5 Groupe critique: un groupe relativement homogène de personnes ayant reçu la plus forte dose attribuable aux rejets des installations en raison de leur lieu d'habitation, de leur style de vie, etc.
} 


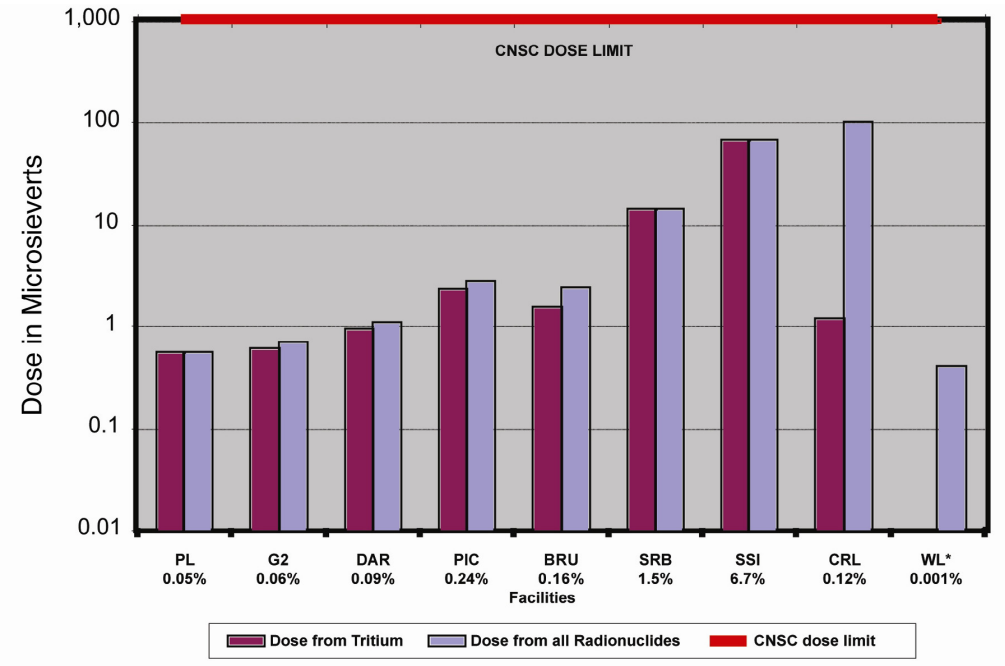

Dose in microsieverts $=$ Dose en microsieverts

CNSC dose limit $=$ Limite de dose de la CCSN

Facilities = Installations

Facilities $=$ Installations
Dose from tritium $=$ Dose (tritium)

Dose from

confondus)
CNSC dose limit $=$ Limite de dose de la CCSN

$\mathrm{PL}=$ Point Lepreau, nouveau Brunswick

$\mathrm{G}_{2}=$ Gentilly -2, Québec

DAR $=$ Darlington, Ontario

PIC $=$ Pickering, Ontario

$\mathrm{BRU}=$ Bruce Power, Ontario

$\mathrm{SRB}=\mathrm{SBR}$ Technologies, Ontario

SSI = Shield Source Inc., Ontario

$\mathrm{CRL}=$ Chalk River Laboratories, Ontario

$\mathrm{WL}=$ Whiteshell Laboratories, Manitoba

Figure 1-Dose de tritium au public en 2006 comparée à la dose de rayonnement de tous les radionucléides et à la limite de dose au public fixée par la CCSN (en abscisse : dose attribuable au tritium en pourcentage de la limite de dose au public).

Tritium dose to the public in 2006 compared to the dose from all radionuclides and to the regulatory limit imposed by CNSC for the public (X axis represents the contribution of tritium (\%) to the regulatory limit dose).

À l'exception des laboratoires de Chalk River, la dose de rayonnement reçue par le public provenait essentiellement du tritium. Dans tous les cas, les doses totales de rayonnement pour toutes les installations, étaient bien en deçà de la limite réglementaire de $1 \mathrm{mSv} . \mathrm{an}^{-1}$. L'exposition du public aux rejets des Laboratoires de Whiteshell (WL) au Manitoba $\left(0,01 \mu \mathrm{Sv} . \mathrm{an}^{-1}\right)$ était trop faible pour être incluse dans la figure 1. 


\subsection{Devenir environnemental du tritium}

Le tritium est typiquement rejeté par les cheminées sous forme de vapeur d'eau tritiée (HTO) ou de gaz élémentaire, l'hydrogène tritié (HT). Lors de son rejet dans l'atmosphère, le processus d'extraction clé du HT comprend l'oxydation photochimique dans l'air et l'oxydation bactérienne dans le sol, ce qui permet la transformation en HTO (Dunstall et al., 1985a, 1985b ; Hart, 2008). Le processus atmosphérique est lent, avec une demi-vie de plus de 5 ans, alors qu'un taux d'oxydation beaucoup plus élevé se produit à l'interface air sol avec une demi-vie variant de 40 minutes à cinq heures (Mishima et Steelec, 2002 ; McFarlane et al., 1978, 1979).

La dynamique du comportement du tritium est en effet régie par des phénomènes physiques et chimiques et par sa durée de séjour variable dans les différents compartiments environnementaux.

L'activité du tritium mesurée dans divers compartiments environnementaux a été comparée aux prédictions effectuées à partir d'un modèle gaussien à moyenne échelle sur les différents secteurs (CSA, 2008). Dans tous les cas, l'activité du tritium prédite par la modélisation dans l'air, dans les précipitations et l'eau du sol, ainsi que dans les eaux souterraines, était supérieure (de 35 à $62 \%$ ) à la valeur mesurée. Il est reconnu que les modèles atmosphériques gaussiens à moyenne échelle ont tendance à être prudents en surestimant la dose annuelle reçue par la population dans le but d'augmenter sa protection. Ces modèles ont été créés pour être utilisés à des fins réglementaires. Pour la modélisation des événements à court terme ou des rejets dans des terrains complexes, des modèles plus complexes sont disponibles (CCSN, 2010a).

La figure 2 illustre l'activité du tritium dans l'air autour des centrales nucléaires.

En général, les programmes de conformité et de surveillance des produits alimentaires visent principalement à mesurer la concentration de HTO dans les produits maraîchers et, de manière plus restreinte, dans le lait et la viande (OBT). Les méthodes courantes d'évaluation de risques et d'estimation des doses au public (CSA N288.1-08) sont fondées sur l'hypothèse que le rapport isotopique $\mathrm{T} / \mathrm{H}$ est identique entre l'eau libre et la matière organique sèche (i.e. que le ratio OBT/HTO, en Bq. $\mathrm{kg}^{-1}$ est égal à 0,8 , compte tenu de l'abondance du carbone dans la matière organique) (CANDU Owners Group, 2008). Cette hypothèse n'a cependant été que partiellement vérifiée par des données sur le terrain et n'a fait l'objet que de peu d'études expérimentales. Des résultats récents de mesure d'OBT dans des zones éloignées des sources de tritium (zones représentatives du bruit de fond naturel) ont révélé des tendances inattendues, montrant des rapports 


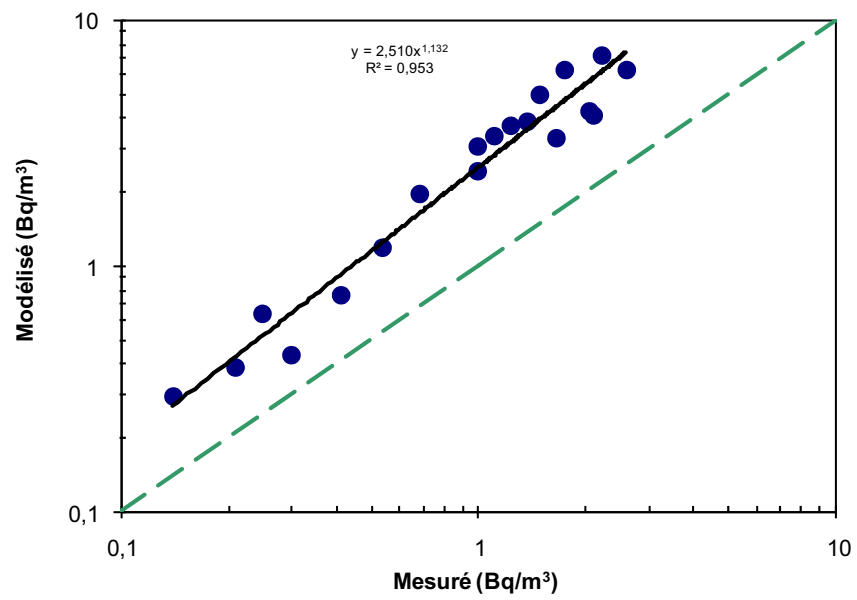

Modélisé $=$ activity estimated from a model Mesuré = measured activity

Figure 2 - Comparaison entre l'activité du tritium mesurée dans des échantillons d'air prélevés autour de centrales nucléaires et les valeurs prédites à l'aide du modèle gaussien.

Comparison of the tritium activity measured in air samples around nuclear power plants with the predicted values obtained using a Gaussian model.

OBT/HTO élevés et contredisant de fait cette importante hypothèse (CANDU Owners Group, 2009).

Les résultats obtenus par la CCSN dans des échantillons prélevés sur des sites de référence qui sont représentatifs du bruit de fond régional en Ontario et en Saskatchewan, sont en accord avec les résultats obtenus en 2009 par le Candu Owners Group. En effet, l'activité en HTO dans les végétaux était similaire à celle de l'eau de pluie et de l'eau du sol, i.e. de l'ordre de 1,2 à 1,8 Bq.L $\mathrm{L}^{-1}$. Toutefois, l'activité de l'eau de combustion de la matière organique dans les végétaux était supérieure à celle de l'eau du sol et atteignait des valeurs de 12,2 Bq.L $\mathrm{L}^{-1}$ en Ontario et de 19,9 Bq.L $\mathrm{L}^{-1}$ en Saskatchewan.

Des études subventionnées par la CCSN ont suggéré que les ratios moyens OBT/HTO, aux environs des sites nucléaires, étaient respectivement de l'ordre de 2 à 3 (des valeurs confirmées par plusieurs données) pour les végétaux et pouvaient atteindre une valeur aussi élevée que 10 pour les produits d'origine animale (une valeur basée sur un nombre limité de données). Il faut comprendre que ces valeurs sont une moyenne de mesures qui incluent des valeurs extrêmes, par exemple, 


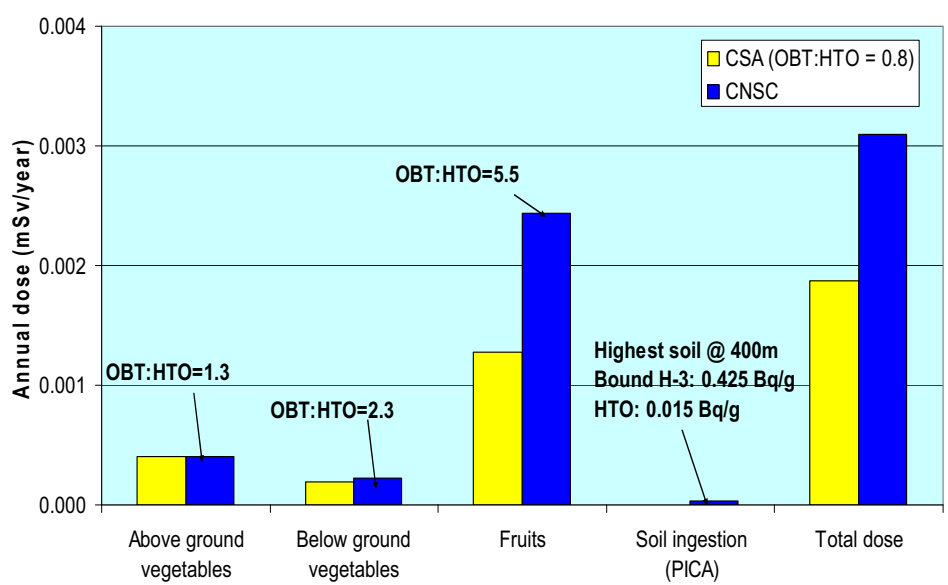

Annual dose $=$ Dose annuelle

$\mathrm{CNSC}=$ Comission canadienne de sûreté nucléaire (CCSN)

CSA $($ Canadian Standard Association $)=$

Association canadienne des normes

Above ground vegetables $=$ Légumes tiges

Below ground vegetables $=$ Légumes racines

Fruits $=$ Fruits

Soil ingestion $=$ Ingestion par le sol

Total dose $=$ Dose totale

Figure 3 - Conséquence dosimétrique d'un rapport OBT/HTO supérieur à la valeur de 0,8 utilisée dans le modèle de la norme CSA N288.1-08.

Influence on dose of an OBT/HTO ratio higher than 0.8 as used in the model of the CSA standard N228.1-08.

certaines mesures effectuées dans un rare mais intéressant contexte (comme celui de la centrale de Darlington ou des installations SRBT et SSI) où les concentrations de HT sont souvent supérieures à celles de HTO. Ce type de situations ayant rarement été considéré, il est possible que les résultats obtenus par la CCSN ne reflètent pas ceux de la littérature scientifique disponible à ce jour. Bien que ces résultats soient beaucoup plus élevés que les valeurs prévues par les modèles physiologiques du comportement de l'HTO et de l'OBT dans les plantes et chez les animaux, ils semblent crédibles. C'est pourquoi la CCSN désire poursuivre ces études afin de valider les données obtenues à ce jour. La figure 3 donne un aperçu des conséquences que peut avoir sur la dose annuelle à la population un rapport OBT/HTO supérieur à la valeur de 0,8 (valeur utilisée dans le modèle de la norme CSA N288.1-08). 
Tableau I

Résumé de 19 études expérimentales en laboratoire sur les effets du tritium selon la dose. Summary of 19 laboratory experiments on the effects of different tritium doses.

\begin{tabular}{lc}
\hline $\begin{array}{l}\text { Dose ou débit de dose minimum pour } \\
\text { induire l'effet }\end{array}$ & $\begin{array}{c}\text { Effet } \\
\text { (animaux laboratoire) }\end{array}$ \\
\hline $5 \mathrm{~Gy}$ & -mortalité \\
$\sim 0,5 \mathrm{~Gy}$ & -promotion cancer \\
$3,7 \mathrm{MBq} / \mathrm{l}$ eau de boisson & -développement embryon/fétus \\
$0,17 \mathrm{MBq} / \mathrm{g} /$ jour & -génétique/reproduction \\
\hline
\end{tabular}

Dose ou débit de dose minimum pour induire l'effet = Dose or minimal dose-rate necessary to induce effects Effet $($ animaux laboratoire $)=$ Effects observed in laboratory animals Mortalité $=$ Mortality

promotion cancer $=$ Cancer promotion

Développement embryon/fétus = Developement of embryo and foetus

Génétique/reproduction $=$ Reproduction/genetics

Eau de boisson $=$ Drinking water

Jour = Day

\subsection{Effets sanitaires du tritium}

Grâce à de nombreuses études expérimentales, tant in vitro qu'in vivo, les effets sanitaires du tritium sont bien connus (Brues et al., 1952 ; Furchner, 1957 ;Yokoro et al., 1986 ; Yamamoto et al., 1990 ; Soloviev et al., 2001 ; Melintescu et al., 2007). Ces études ont révélé que le rayonnement du tritium, comme d'autres types de rayonnement, peut nuire au développement de l'embryon et du fœtus (Dekaban, 1968 ; Brenner et al., 2003, BEIR III, 1980 ; Straume et Carsten, 1993 ; BEIR VII, 2007). Il peut aussi avoir des effets génétiques et sur l'appareil reproducteur ainsi qu'entraîner la mort cellulaire (Straume et Carsten, 1993), mais cela n'a pu être démontré qu'à des doses des millions de fois supérieures à celles auxquelles l'activité industrielle pourrait exposer la population. Il peut induire le cancer chez les animaux dans certaines conditions expérimentales (Yamamoto et al., 1995 ; Straume, 1993), mais là encore, uniquement à fortes doses. La quantité de tritium requise pour causer ces effets est de l'ordre du gigabecquerel $\left(10^{9}\right)$ par gramme de poids corporel avec des doses supérieures à $500 \mathrm{mSv}$ (Tab. I). À titre de référence, l'exposition professionnelle au tritium des travailleurs canadiens en 2006 était inférieure à $1 \mathrm{mSv}$ par année alors que la limite réglementaire pour ces mêmes travailleurs est de $50 \mathrm{mSv}$ par année ou de $100 \mathrm{mSv}$ pour une période de cinq ans. De son côté, la dose pour la population attribuable aux rejets de tritium dans l'environnement est généralement inférieure à $0.067 \mathrm{mSv}$ par année bien en deçà de la limite réglementaire fixée à $1 \mathrm{mSv}$ par année, comme illustré sur la figure 1 .

De nombreuses études expérimentales ont été menées afin de caractériser la valeur de l'efficacité biologique relative du tritium (EBR) (Straume et Carsten, 
1993 ; Little et Lambert, 2008). Les effets biologiques des rayonnements sont variables en fonction de leur nature et de leur énergie. Des facteurs de corrections sont utilisés pour permettre de traduire l'énergie absorbée par un tissu (en J.kg ${ }^{-1}$ ou Gy) en dose équivalente (Sv). Cette dernière grandeur permet de cumuler les effets similaires des différents types de rayonnements. L'EBR permet d'établir la meilleure estimation des doses individuelles lors d'études épidémiologique. Elle est aussi utile dans les études rétrospectives lorsqu'une mesure plus précise de la dose est nécessaire et dans les cas d'expositions suffisamment sérieuses pour entraîner un traitement médical (Kocher et al., 2005).

Les différents résultats de calcul d'EBR sont pris en compte pour définir un facteur de pondération global pour les différents rayonnements. Le choix d'une valeur unique pour représenter l'EBR du tritium a fait l'objet de plusieurs débats scientifiques au cours des dernières années (HPA, 2007). La Commission internationale de protection radiologique (CIPR) utilise un fateur de pondération $\left(\mathrm{W}_{\mathrm{R}}\right)$ de 1 pour tous les rayonnements à faible transfert linéique d'énergie (TLE) comme le rayonnement bêta émis par le tritium, alors que de nombreuses études ont indiqué que l'EBR du tritium à induire le cancer serait deux fois plus importante que celle des autres rayonnements de faible TLE. Il existe dans la littérature plus de 50 estimations différentes de l'EBR du tritium. Toutefois, il existe un degré important de variation et d'incertitude dans les données radio-biologiques disponibles, d'où la difficulté de choisir une valeur nominale unique de facteur de pondération à des fins de radioprotection. L'une des principales sources de ces variations réside dans le fait qu'il existe plus d'un rayonnement de référence pour calculer l'EBR. En général, deux types de rayonnement de référence sont utilisés, soit les rayons $\mathrm{X}$ et les rayons gamma et ils conduisent à des EBR différentes.

Les études visant à déterminer une EBR unique pour le tritium indiquent que :

- lorsque les rayons $X$ sont utilisés comme référence, l'EBR est d'environ 1,4 (Little et Lambert, 2008) ;

- lorsque le rayonnement gamma est utilisé comme référence, l'EBR est d'environ 2,2.

La Publication 92 (ICRP, 2003) privilégie le choix du rayonnement gamma comme référence en s'appuyant sur le fait que les principes de radioprotection reposent principalement sur les données d'études des survivants de la bombe atomique et que ces survivants ont presqu'exclusivement été exposés à des rayons gamma. De plus, le rayonnement gamma est celui qui est le plus étudié dans les expériences portant sur les effets des expositions chroniques au rayonnement et il est aussi le principal rayonnement auquel les travailleurs du secteur nucléaire sont exposés. 
Tenant compte des données expérimentales disponibles, plusieurs auteurs estiment que l'utilisation d'une valeur nominale unique de facteur de pondération de 1 par la CIPR sous-estime le risque sanitaire du tritium (Nikjoo et al., 2001, 2002a, 2002b ; Moiseenko et al., 2001a, 2001b ; Hill, 2004 ; Krumrey et al., 2004).

Toutefois, lorsqu'il s'agit d'estimer les effets sanitaires de type aléatoire (comme le cancer) liés à l'exposition au rayonnement, les études expérimentales en laboratoire ont leur limite. Les études épidémiologiques fondées sur des données de qualité concernant l'exposition aux rayonnements demeurent la meilleure source d'information pour évaluer leurs effets réels sur la santé des humains.

Un grand nombre d'études épidémiologiques sur des travailleurs exposés au rayonnement du tritium, sur leurs descendants ainsi que sur des citoyens vivant à proximité d'installations nucléaires, ont été évaluées au cours de ce projet (CCSN, 2010b). Dans l'ensemble, très peu d'études contenaient suffisamment de renseignements pour estimer directement les risques sanitaires associés à l'exposition au tritium. En effet plusieurs études comportaient des lacunes importantes, par exemple, un manque de données sur les doses spécifiques résultant de l'exposition au tritium, de faibles doses de rayonnement total, un petit nombre de cas d'exposition et une puissance statistique insuffisante.

\subsection{1. Études épidémiologiques sur des travailleurs exposés au rayonnement du tritium}

La principale conclusion qui peut être tirée de la revue des études épidémiologiques menées sur les travailleurs du secteur nucléaire dans le cadre de ce projet est : l'impossibilité de faire une inférence directe entre l'augmentation du risque de cancer et l'exposition au tritium des travailleurs de l'United Kingdom Atomic Energy Authority (UKAEA) (Beral et al., 1985 ; Fraser et al., 1993 ; Atkinson et al., 2004, 2007), de l'Atomic Weapons Establishment (AWE) (Beral et al., 1988 ; Johnson et al., 1999) ou des sites de Sellafield (Smith et Douglas, 1986 ; Douglas et al., 1994 ; Omar et al., 1999), Capenhurst (McGeoghegan et Binks, 2000) ou Chapelcross (McGeoghegan et Binks, 2001), en raison de l'absence de données dosimétriques portant expressément sur le tritium. Par contre, il a été mis en évidence que, si les données dosimétriques concernant le tritium des travailleurs du site de Capenhurst, lesquels auraient subi une exposition considérable au tritium, pouvaient être rendues disponibles, cette cohorte fournirait de précieux renseignements. Il a aussi été démontré qu'il était difficile de tirer des conclusions sur les risques associés au tritium à partir des données des études canadiennes et américaines. Même si les doses de tritium avaient été mesurées et intégrées aux évaluations, peu d'analyses avaient porté directement sur les effets de l'exposition au tritium. 
Le projet a aussi révélé que si le problème des registres historiques de données (Ashmore et al., 2010) pour la cohorte des travailleurs canadiens du secteur nucléaire était résolu, cette dernière pourrait constituer une précieuse source d'information pour l'évaluation du risque associé au tritium. Il convient de rappeler qu'il existe des incertitudes substantielles dans l'estimation du risque de la cohorte des travailleurs canadiens dans l'analyse de la Commission Internationale de Recherche sur le Cancer (CIRC) qui incluait les travailleurs de 15 pays (UNSCEAR, 2008). Le risque estimé dans ces études était en effet très faible, voire nul, pour la leucémie et les cancers solides, pour tous les travailleurs des centrales nucléaires exposés à des produits radioactifs, dont le tritium, sauf pour les travailleurs canadiens.

La revue des quatre études américaines considérées dans le cadre du projet a démontré une corrélation positive entre la mortalité par leucémie et une exposition à un rayonnement ionisant de faible intensité en milieu de travail (Cragle et al., 1988, 1998 ; Schubauer-Berrigan et al., 2007 ; Richardson et Wing, 2007). Ici encore, il est difficile de tirer une quelconque conclusion quant au risque associé à une exposition au tritium seul. Même si, dans ces études, les doses de tritium avaient été mesurées et intégrées dans les doses auxquelles les travailleurs étaient exposés, peu d'analyses ont porté sur les effets de l'exposition au tritium uniquement. Il serait bien de noter qu'étant donné l'importance de l'exposition au tritium des travailleurs du site de Savannah River (SRS), cette cohorte pourrait éventuellement fournir de précieux renseignements sur le risque sanitaire attribué au tritium.

Ce sont les études sur les travailleurs exposés au rayonnement qui ont fourni à ce jour les meilleures données disponibles sur le risque sanitaire du tritium. Toutefois, quelques études seulement faisaient état de mesures directes de l'exposition au tritium et un plus petit nombre encore avaient évalué les risques sanitaires attribuables au tritium seulement. Il est difficile d'isoler la part de risque attribuable au tritium seulement, puisque sa contribution à l'exposition totale est très faible par rapport aux autres sources de rayonnement.

\subsection{2. Études épidémiologies sur les descendants des travailleurs du secteur nucléaire et sur les citoyens vivant à proximité d'installations nucléaires}

Plusieurs études écologiques qui ont évalué les effets potentiels sur la santé des rejets de tritium dans l'environnement sur les populations habitant près des installations nucléaires ont été revues dans le cadre du projet (CCSN, 2010b). Ces études par contre ne comportaient aucune évaluation individuelle de l'exposition au tritium (ou d'autres facteurs de risque). En raison des limites associées à ce type d'études (UNSCEAR, 2008), il a été conclu qu'il n'était pas possible d'établir une inférence entre l'exposition au tritium et ses effets potentiels sur la santé. Il est, par contre 
possible, d'avancer que l'existence d'effets nocifs sur la santé (incluant ceux qui seraient liés aux rejets de tritium) serait improbable ; les niveaux d'exposition au rayonnement provenant des installations étudiées étant extrêmement faibles (Sever et al., 1988a, 1988b ; Jablon et al., 1991 ; Grosche et al., 1999 ; Evrad et al., 2006 ; Durham Region Health Department, 2007 ; CCSN, 2009).

Les études cas-témoins récentes «Kinderkrebs in der Umgebung von Kernkraftwerken » $(\mathrm{KiKK})$ menées en Allemagne sur la leucémie infantile (Kaatsch et Mergenthaler, 2008 ; Spix et al., 2008) ont reçu beaucoup d'attention. Leur revue et la revue d'autres études semblables en France (Laurier et Bard, 1999 ; Laurier et al., 2002, 2008) et en Grande-Bretagne (COMARE, 2006, 2011) n'ont pas permis de déterminer à ce jour l'existence d'un lien de causalité entre un facteur de risque chimique ou physique et le risque observé de leucémie infantile chez les enfants de moins de 5 ans habitant dans un rayon de $5 \mathrm{~km}$ des centrales nucléaires tel que l'avait suggéré l'étude KiKK. Il n'en reste pas moins que le risque observé demeure inexpliqué.

De plus, les revues des cas de leucémie infantile à proximité des installations nucléaires par Laurier et al. (Laurier et Bard, 1999 ; Laurier et al., 2002, 2008) n'ont confirmé l'existence que de trois grappes de cas de leucémie ayant persisté dans le temps, soit aux environs de Sellafield, Dounreay et Krümmel. Ces études n'ont pu mettre en évidence aucun lien de causalité entre la leucémie chez l'enfant et l'exposition environnementale à la radioactivité près des installations nucléaires ou l'exposition parentale avant la conception. Deux études canadiennes (McLaughlin et al., 1992, 1993) ont évoqué la possibilité d'établir des inférences directes entre le risque de leucémie infantile et l'incidence d'anomalies congénitales reliées à l'exposition paternelle au tritium avant la conception. Cependant, le nombre de cas et de témoins dans le groupe exposé était trop faible, conférant ainsi à l'étude une puissance statistique insuffisante.

L'ensemble des études épidémiologiques revues dans le cadre du projet de la CCSN ont démontré l'absence d'un excès de risque statistiquement significatif pour des niveaux d'exposition inférieurs ou égaux à 100 mSv. Malgré les lacunes des études identifiées ci-dessus, il s'avère possible, suite au projet, de supposer que tout risque attribuable au tritium seul serait négligeable et qu'il serait difficile de l'isoler des autres risques similaires sur la santé dans la population.

\section{Conclusions et recommandations}

Les données environnementales et les résultats des expositions professionnelles, compilés en 2006 dans le cadre du projet sur le tritium, indiquent que le tritium, généré par l'opération des centrales nucléaires et des installations de traitement du 
tritium, ne représentait qu'une infime fraction des faibles doses auxquelles sont exposés le public et les travailleurs du secteur nucléaire, soit entre 0,01 et $14,5 \mu \mathrm{Sv}$, bien en deçà de la limite de dose réglementaire pour la population. Les doses de rayonnement dues au tritium sont en effet de plusieurs ordres de grandeur inférieurs aux doses reconnues pour causer des effets sur la santé.

Comme démontré par la revue exhaustive des connaissances scientifiques actuelles, effectuée dans le cadre du projet sur le tritium de la CCSN, le devenir du tritium dans l'environnement, après son rejet dans l'atmosphère par les cheminées des installations nucléaires, a suscité beaucoup d'intérêt et est assez bien cerné.

Il existe néanmoins des imprécisions dans la modélisation des transferts du tritium dans l'environnement. Ces imprécisions se situent surtout dans les mesures du rapport OBT/HTO. Il existe aussi des incertitudes sur l'impact qu'ont les rejets types des installations de traitement du tritium sur la formation et la rétention de l'OBT dans les sols et les plantes. Avant que ce projet ne soit initié, très peu d'études sur le tritium dans l'environnement avaient été menées autour des installations de traitement du tritium. En effet, ces installations rejettent principalement de l'HT, alors que les autres installations nucléaires (par exemple le réacteur CANDU) rejettent essentiellement, sinon uniquement, de l'HTO comme mentionné plus-haut.

Dans le cadre de ce projet, les résultats d'OBT obtenus autour des installations qui possèdent des réservoirs ou des sources et des régimes de rejet du tritium différents de ceux du CANDU, comme la centrale de Darlington ou les deux installations de traitement du tritium SRBT et SSI, ont révélé des rapports OBT/HTO plus élevés dans les sols et dans certains produits alimentaires que ceux qui sont prévus par les modèles (CCSN, 2010c). Les travaux de la CCSN ont aussi démontré que non seulement les rapports OBT/HTO moyens pouvaient être plus élevés que prévu mais aussi qu'ils étaient plus variables que ne le laissaient présumer les modèles courants de comportement du tritium. En se basant sur les mesures d'HTO et d'OBT dans les produits des jardins potagers, la dose annuelle, reçue par les résidants vivant autour de l'installation de traitement du tritium SRBT et découlant de la consommation de produits locaux, était inférieure à 0,004 $\mathrm{mSv}$, soit une infime fraction de la limite de dose réglementaire canadienne de 1 $\mathrm{mSv}$ pour la population et seulement légèrement supérieure à la dose attribuable aux niveaux du bruit de fond régional pour le tritium.

Dans le contexte canadien actuel, lequel est caractérisé par de faibles rejets de tritium dans l'atmosphère par les installations nucléaires, les concentrations relativement élevées d'OBT mesurées dans un petit nombre de produits alimentaires cultivés localement n'ont pas de conséquences sur la santé, parce qu'elles génèrent 
des doses qui demeurent très faibles. Jusqu'à ce que des études additionnelles puissent confirmer ces résultats préliminaires, le personnel de la CCSN recommande :

- qu'à court terme, les titulaires de permis pour les installations qui rejettent du tritium tiennent compte dans leur calcul des doses au public des conséquences de l'utilisation d'un rapport OBT/HTO maximum de 3 dans les produits maraîchers et le fourrage cultivés localement, et d'un rapport OBT/HTO maximum de 10 dans les produits dérivés d'animaux d'élevage locaux (viande, lait et oeufs) ;

- de poursuivre son programme de recherche dans ce domaine pour mieux comprendre les mécanismes responsables des rapports OBT/HTO observés ;

- de collaborer avec d'autres organismes nationaux et internationaux pour élaborer des procédures normalisées d'analyse de l'OBT dans diverses matrices environnementales.

La revue de la littérature disponible à ce jour sur les effets sanitaires du tritium a permis à la CCSN de faire les constatations suivantes :

- selon les données des études épidémiologiques et des études en laboratoire, la probabilité que l'exposition au tritium, aux niveaux actuellement observés au Canada, ait des effets néfastes sur la santé est très faible ;

- les résultats de plus de 50 études expérimentales concernant la détermination d'un facteur de pondération unique pour le tritium confirment que les particules bêta émises par le tritium ont une efficacité à produire des effets biologiques qui est environ 1,4 fois celle des rayons $\mathrm{X}$ de $250 \mathrm{kV}_{\mathrm{p}}$ et environ 2,2 fois celle des rayons gamma ;

- le recours à un facteur de pondération de valeur 1 , prévu par la Commission internationale de protection radiologique (CIPR) et utilisé dans le cadre actuel de radioprotection, n'a pas entraîné de diminution de la protection offerte aux travailleurs ou aux membres du public. En effet, la mise en vigueur de mesures d'optimisation s'est traduite par des expositions au tritium qui sont très faibles et bien inférieures aux limites réglementaires.

Dans un contexte de radioprotection, ces constatations indiquent que le cadre actuel de réglementation canadien permet de contrôler efficacement l'exposition au tritium. Néanmoins, en tenant compte de la revue des données expérimentales sur l'EBR, du manque d'études épidémiologiques robustes spécifiques au tritium et des inquiétudes exprimées par le public, le personnel de la CCSN a recommandé la poursuite de son programme de recherche en épidémiologie et plus particulièrement :

- la possible initiation d'une étude de cohortes internationales sur l'incidence et la mortalité dues au cancer chez les travailleurs du secteur nucléaire exposés au tritium ; 
- la conduite d'études de cas-témoins sur les cancers et les anomalies congénitales dues à l'exposition in utero des enfants des travailleurs canadiens du secteur nucléaire exposés au tritium (ces études pourraient englober quelques centaines de cas-témoins en Ontario au Québec et au Nouveau Brunswick) ;

- la mise sur pied d'un groupe de travail sur le tritium qui serait formé de représentants d'organismes de santé publique, d'universités et d'organisations non gouvernementale. Ce groupe aurait le mandat de donner son avis sur la conception des études nationales et internationales proposées ainsi que d'en examiner les résultats ;

- l'examen par le groupe de travail de la question du facteur de pondération des rayonnements à faible transfert linéique d'énergie, comme celui du tritium.

Suite à ce projet, il a été possible de conclure que les dispositions prises, en ce qui concerne le tritium, pour protéger la santé et la sécurité des canadiens dans le cadre des mécanismes réglementaires existants, sont adéquates. Les pratiques canadiennes de manipulation et de contrôle du tritium sont comparables à celles qui existent pour le même type d'installations à l'étranger. Le contrôle exercé au moyen des divers mécanismes utilisés est efficace.

Il a aussi été possible de conclure que, dans le cadre réglementaire existant, les doses au public ou aux travailleurs du secteur nucléaire, attribuables à la gestion du tritium dans les installations nucléaires, sont très faibles et largement inférieures aux limites réglementaires. En effet, aucune incertitude identifiée lors de notre étude n'est de nature à compromettre la santé du public et des travailleurs. Toutefois, la poursuite des activités de recherche identifiées ci-dessus permettrait à la CCSN de réglementer le tritium en se basant sur des connaissances scientifiques encore plus robustes.

\section{RÉFÉRENCES}

Ashmore P.J., Gentner N.E., Osborne R.V. (2010) Incomplete data on the Canadian cohort may have affected the results of the study by the International Agency for Research on Cancer on the radiogenic cancer risk among nuclear industry workers in 15 countries, J. Rad. Prot. 30, 115120.

Atkinson W.D., Law D.V., Bromley K.J., Inskip H.M. (2004) Mortality of employees of the United Kingdom Atomic Energy Authority, 1946-97, Occup. Environ. Med. 61, 577-585.

Atkinson W.D., Law D.V., Bromley K.J. (2007) A decline in mortality from prostate cancer in the UK Atomic Energy Authority workforce, J. Radiol. Prot. 27, 437-445.

Autorité de sûreté nucléaire (ASN) (2010) Livre Blanc du tritium. Groupes de réflexion menés de mai 2008 à avril 2010 sous l'égide de l'ASN, Paris, France, 278 p.

BEIR III. National Research Council (NRC) (1980) The effects on populations of exposure to low levels of ionizing radiation. The Committee on the Biological Effects of Ionizing Radiations Washington, DC: National Academy Press.

BEIR VII. National Research Council (NRC) (2007) Health Risks from Exposure to Low Levels of Ionizing Radiation: Phase 2. Board on Radiation Effects Research. The Committee on the Biological Effects of Ionizing Radiations Washington, DC: National Academies Press. 
Beral V., Inskip H., Fraser P., Booth M., Coleman D., Rose G. (1985) Mortality of employees of the United Kingdom Atomic Energy Authority, 1946-1979, Brit. Med. J. 291, 440-447.

Beral V., Fraser P., Carpenter L., Booth M., Brown A., Rose G. (1988) Mortality of employees of the Atomic Weapons Establishment, 1951-82, Brit. Med. J. 297, 757-770.

Brenner D.J., Doll R., Goodhead D.T., Hall E.J., Land C.E., Little J.B., Lubin J.H., Preston D.L., Preston R.J., Puskin J.R., Ron E., Sachs R.K., Samet J.M, Setlow, R.B., Zaider M. (2003) Cancer risks attributable to low doses of ionizing radiation: Assessing what we really know, Proc. Nat. Acad. Sci. USA 100, 13761-13766.

Brues A.M., Stroud A.N., Rietz L. (1952) Toxicity of tritium oxide to mice, Proc. Soc. Exp. Biol. Med. 79, $174-176$.

Canadian Standard Association (CSA) (2008) N288.1-08 - Guidelines for calculating derived release limits for radioactive material in airborne and liquid effluents for normal operation of nuclear facilities, publiée par CSA, $354 \mathrm{p}$.

CANDU Owners Group (2008) OBT/HTO ratios in plants, COG-06-3053-R1.

CANDU Owners Group (2009) Vegetable OBT/HTO ratios in background areas of Ontario, COG-093015.

Commission canadienne de sûreté nucléaire (CCSN) (2009) Le rejet de tritium et ses conséquences radiologiques au Canada en 2006, INFO-0793, Ministère des travaux publiques er services gouvernementaux Canada, numéro de catalogue CC172-52/2009E-PDF, ISBN 978-1-10013930-2. Disponible à :

http://www.nuclearsafety.gc.ca/pubs_catalogue/uploads_fre/CNSC_Release_and_Dose_fr.pdf.

Commission canadienne de sûreté nucléaire (CCSN) (2010a) Étude sur le devenir environnemental du tritium dans l'atmosphère, Volet du projet d'études sur le tritium, INFO-0792, Ministère des travaux publiques er services gouvernementaux Canada, numéro de catalogue: CC17251/2009F-PDF, ISBN 978-1-100-92798-5. Disponible à :

http://www.nuclearsafety.gc.ca/pubs_catalogue/uploads_fre/Investigation_of_Environmental_ Fate_of_Tritium_in_the_Atmosphere_INFO-0792_f.pdf.

Commission canadienne de sûreté nucléaire (CCSN) (2010b) Tritium : Effets sur la santé, dosimétrie et radioprotection, Volet du projet d'études sur le tritium, INFO-0799, Ministre de Travaux publics et Services gouvernementaux Canada, numéro de catalogue : CC172-58/2010F-PDF, ISBN 978-1-100-94408-1. Disponible à :

http://www.nuclearsafety.gc.ca/pubs_catalogue/uploads_fre/CNSC_Health_Effects_Fre-web.pdf.

Commission canadienne de sûreté nucléaire (CCSN) (2010c) Taux de tritium dans les produits maraîchers de Pembroke en 2007 et dose à la population. Volet du projet d'études sur le tritium, INFO-0798, Ministère des travaux publiques er services gouvernementaux Canada, numéro de catalogue : CC172-57/2010F-PDF, ISBN 978-1-100-94406-7. Disponible à : http://www.nuclearsafety.gc.ca/pubs_catalogue/uploads_fre/CNSC_Garden_Produce_Fre-web.pdf.

Committee on Medical Aspects of Radiation in the Environment (COMARE) (2006) Eleventh Report. The distribution of childhood leukaemia and other childhood cancer in Great Britain 1969-1993. Disponible à : http://www.comare.org.uk/comare_docs.htm.

Committee on Medical Aspects of Radiation in the Environment (COMARE) (2011) Fourteenth Report. Further consideration of the incidence of childhood leukaemia around nuclear power plants in Great Britain. Disponible à : http://www.comare.org.uk/comare_docs.htm.

Cragle D.L., McLain R.W., Qualters J.R., Hickey J.L.S., Wilkinson G.S., Tankersley W.G., Lushbaugh C.C. (1988) Mortality among workers at a nuclear fuels production facility, Am. J. Indus. Med. 14, 379-401.

Cragle D.L., Watkins J.P., Robertson-DeMers K. (1998) Mortality among workers at the Savannah River nuclear fuels production facility, ASA 1998 proceedings of the section on statistics in epidemiology, Alexandria, VA American Statistical Association, pp. 83-87. URL: http://www.orau.gov/ehsd/EpiProceedings-jj-9-30-98.doc. 
Dekaban A.S. (1968) Abnormalities in children exposed to x-radiation during various stages of gestation: tentative timetable of radiation injury to the human fetus, Int. J. Nucl. Med. 9, 471477.

Douglas A.J., Omar R.Z., Smith P.G. (1994) Cancer mortality and morbidity among workers at the Sellafield plant of British Nuclear Fuels, Brit. J. Canc. 70, 1232-1243.

Dunstall T.G., Ogram G.L., Spencer F.S. (1985a) Elemental tritium deposition and conversion in the terrestrial environment, Programme technique canadien des combustibles de fusion, rapport CFFTP-G-85035

Dunstall T.G., Ogram G.L., Spencer F.S. (1985b) Elemental tritium deposition and conversion in the terrestrial environment, Fusion Technol. 8, 2551-2556.

Durham Region Health Department (2007) Radiation and Health in Durham Region, Durham Region, Ontario, Canada.

Evrard A-S., Hemon D., Morin A., Laurier D., Tirmarche M., Backe J.-C., Cartier M. Clavel J. (2006) Childhood leukaemia incidence around French nuclear installations using geographic zoning based on gaseous discharge dose estimates, BJC 94, 1342-1347.

Fraser P., Carpenter L., Moconochie N., Higgins C., Booth M., Beral V. (1993) Cancer mortality and morbidity in employees of the United Kingdom Atomic Energy Authority, 1946-86, Brit. J. Canc. 67, 615-624.

Furchner J.E. (1957) Relative Biological Effectiveness of Tritium Beta-Particles and Cobalt 60 Gamma-Rays Measured by Lethality in CF1 Mice, Rad. Res. 6, 483-490.

Galeriu D., Heling R., Melintescu A. (2005) The dynamics of tritium including OBT in the aquatic food chain, Fusion Sci. Technol. 48, 779-782.

Greenpeace Report (2007) Tritium Hazard Report: Pollution and Radiation Risk from Canadian Nuclear Facilities. Un rapport préparé pour Greenpeace par I. Fairlie. http://www.greenpeace.org/raw/content/canada/en/documents-and-links/publications/tritiumhazard-report-pollu.pdf.

Grosche B., Lackland D., Mohr L., Dunbar J., Nicholas J., Burkart W., Hoel D. (1999) Leukaemia in the vicinity of two tritium-releasing nuclear facilities: a comparison of the Kruemmel Site, Germany, and the Savannah River Site, South Carolina, USA, J. Radiol. Prot. 19, 243-252.

Hart D. (2008) Derived release limits guidance, Rapport technique du Groupe des propriétaires de CANDU, COG-06-3090.

Health Protection Agency (HPA) (2007) Review of risks from tritium with particular attention to tritiated water and organic compounds containing tritium. Report of AGIR subgroup on tritium risks, UK advisory group on ionizing radiation. http://www.hpa.org.uk/radiation/advisory_groups/agir/index.htm.

Hill M.A. (2004) The variation of biological effectiveness of X-rays and gamma rays with energy, $\mathrm{Rad}$. Prot. Dos. 112, 471-481.

ICRP Publication 92 (2003) Relative Biological Effectiveness (RBE), Quality Factor (Q), and Radiation Weighting Factor ( $\mathrm{W}_{\mathrm{R}}$ ). ICRP Publication 92, Ann ICRP 33, 1-121.

Jablon S., Hrubec Z., Boice J.D. Jr. (1991) Cancer in Populations Living Near Nuclear Facilities. A survey of mortality nationwide and incidence in two States, JAMA 265, 1403-1408.

Johnson P., Atkinson W.D., Nicholls J.L. (1999) Updated analysis of mortality in workers at UK atomic weapons establishments, Proceedings of the SRP Sixth International Symposium: Achievements \& Challenges: Advancing Radiation Protection into the 21st Century.

Kaatsch P., Mergenthaler A. (2008) Incidence, time trends and regional variation of childhood leukaemia in Germany and Europe, Rad. Prot. Dos. 132, 107-113.

Kocher D.C., Apostoaei A.I., Hoffman FO. (2005) Radiation effectiveness factors for use in calculating probability of causation of radiogenic cancers, Health Phys. 89, 3-32. 
Krumrey M., Ulm G., Schmidt E. (2004) Dicentric chromosomes in monolayers of human lymphocytes produced by monochromatized syncrhotron radiation with photon energies from $1.83 \mathrm{keV}$ to $17.4 \mathrm{keV}$, Rad. Environ. Biophys. 43, 1-6.

Laurier D., Bard D. (1999) Epidemiologic studies of leukemia among persons under 25 years of age living near nuclear sites, Epidemiol. Rev. 21, 188-206.

Laurier D., Grosche B., Hall P. (2002) Risk of childhood leukaemia in the vicinity of nuclear installations - findings and recent controversies, Acta Oncol. 41,14-24.

Laurier D., Jacob S., Bernier M.O., Leuraud K., Metz C., Samson E., Laloi P. (2008) Epidemiological Studies of Leukaemia in Children and Young Adults around Nuclear Facilities: A Critical Review, Rad. Prot. Dos. 132, 182-190.

Little M.P., Lambert B.E. (2008) Systematic review of experimental studies of relative biological effectiveness of tritium, Rad. Environ. Biophys. 47, 71-93.

McFarlane J.C., Rodgers R.D., Bradley D.V. Jr. (1978) Environmental tritium oxidation in surface soil, Environ. Sci. Technol. 12, 590-593.

McFarlane J.C., Rodgers R.D., Bradley D.V. Jr. (1979) Tritium oxidation in surface soils. A survey of soils near five fuel reprocessing plants, Environ. Sci. Technol. 13, 607-608.

McGeoghegan D., Binks K. (2000) The mortality and cancer morbidity experience of workers at the Capenhurst uranium enrichment facility, 1946-95, J. Radiol. Prot. 20, 381-401.

McGeoghegan D., Binks K. (2001) The mortality and cancer morbidity experience of employees at the Chapelcross plant of British Nuclear Fuels plc, 1955-95, J. Radiol. Prot. 21, 221-250.

McLaughlin J., Anderson T.W., Clarke E.A., King W. (1992) Occupational exposure of fathers to ionizing radiation and the risk of leukaemia in offspring - a case-control study (AECB project no 7.157.1). Report INFO-0424. Atomic Energy Control Board, Ottawa, Canada.

McLaughlin J.R., King W.D., Anderson T.W., Clarke E.A., Ashmore J.P. (1993) Paternal radiation exposure and leukaemia in offspring: the Ontario case-control study, BMJ 307, 959-966.

Melintescu A., Galeriu D., Takeda H. (2007) Reassessment of tritium dose coefficients for the general public, Rad. Prot. Dos. 127, 153-157.

Mishima J., Steelec C.M. (2002) Oxidation of Tritium Gas under Accident and Transport Conditions. Rapport du DOE, LA-UR-02-3803.

Moiseenko V.V., Hamm R.N., Waker A.J., Prestwich W.V. (2001a) Calculation of radiationinduced DNA damage from photons and tritium beta-particles. Part I: Model formulation and basic results, Rad. Environ. Biophys. 40, 23-31.

Moiseenko V.V., Waker A.J., Hamm R.N., Prestwich W.V. (2001b) Calculation of radiationinduced DNA damage from photons and tritium beta-particles Part II: Tritium RBE and damage complexity, Rad. Environ. Biophys. 40, 33-38.

Nikjoo H., O’Neil P., Wilson W.E., Goodhead D.T. (2001) Computational approach for determining the spectrum of DNA damage induced by ionizing radiation, Rad. Res. 156, 577-583.

Nikjoo H., Bolton C.E., Watanabe R., Terrissol M., O’Neill P., Goodhead D.T. (2002a) Modeling of DNA Damage Induced by Energetic Electrons (100 eV to $100 \mathrm{keV})$, Rad. Prot. Dos. 99, 77-80.

Nikjoo H., Goorley T., Fulford J., Takakura K., Ito T. (2002b) Quantitative Analysis of Energetics of DNA Damage, Rad. Prot. Dos. 99, 91-98.

Omar R.Z., Barber J.A., Smith P.G. (1999) Cancer mortality and morbidity among plutonium workers at the Sellafield plant of British Nuclear Fuels, Brit. J. Canc. 79, 1288-1301.

Richardson D.B., Wing S. (2007) Leukemia mortality among workers at the Savannah River Site, Am. J. Epidemiol. 166, 1015-1022.

Schubauer-Berrigan M.K., Daniels R.D., Fleming D.A., Markey A.M., Couch J.R., Ahrenholz S.H., Burphy J.S., Anderson J.L., Tseng C.-Y. (2007) Risk of chronic myeloid and acute leukemia mortality after exposure to ionizing radiation among workers at four U.S. nuclear weapons facilities and a nuclear naval shipyard, Rad. Res. 167, 222-232. 
Sever L.E., Gilbert E.S., Hessol N.A., McIntyre J.M. (1988a) A case-control study of congenital malformations and occupational exposure to low-level ionizing radiation, Am. J. Epidemiol. 127, 226-242.

Sever L.E., Hessol N.A., Gilbert E.S., McIntyre J.M. (1988b) The Prevalence at Birth of Congenital Malformations in Communities near the Hanford Site, Am. J. Epidemiol. 127, 243-254.

Smith P.G., Douglas A.J. (1986) Mortality of workers at the Sellafield plant of British Nuclear Fuels, Brit. Med. J. (Clin Res Edition) 293, 845-854.

Soloviev V., Ilyin L.A., Baranov A.E. et al. (2001) Medical Management of Radiation Accidents, Second edition (I.A. Gusev, A.K. Guskova and F.A. Mettler, Eds.) CRC Press, Boca Raton, pp. 46 et 158.

Spix C., Schmiedel S., Kaatsch P., Schulze-Rath R., Blettner M. (2008) Case-control study on childhood cancer in the vicinity of nuclear power plants in Germany 1980-2003, Eur. J. Canc. 44, 275-244.

Straume T. (1993) Tritium Risk Assessment, Health Phys. 65, 673-682.

Straume T., Carsten A.L. (1993) Tritium radiobiology and relative biological effectiveness, Health Phys. 65, 657-672.

United Nations Scientific Committee on the Effects of Atomic Radiation (UNSCEAR) (2008) United Nations. Effects of Ionizing Radiation, Rapport de 2006 à l'Assemblée générale et ses annexes scientifiques, Nations Unies, New York.

Weiss W., Bullacher J., Roether W. (1979) Evidence of pulsed discharges of tritium from nuclear energy installations in Central Europe precipitation, dans : Behaviour of Tritium in the Environment. IAEA-SM-232/18, pp. 17-30.

World Health Organization (WHO) (1983) Environmental Health Criteria 25, Geneva, Switzerland, WHO.

Yamamoto O., Yokoro T., Seyama A., Kinomura T. (1990) HTO Oral Administration in Mice. I: Threshold Dose Rate for Haematopoietic Death, Int. J. Rad. Biol. 57, 543-549.

Yamamoto O., Seyama T., Jo T., Terato H., Saito T., Kinomura A. (1995) Oral Administration of Tritiated Water (HTO) in Mouse. II. Tumour Development: I/II/III, Int. J. Rad. Biol. 68, 47-54.

Yokoro K., Yamamoto O., Seyama T., Kinomura A. (1986) Acute and Chronic Effects of Tritiated Water in Mice with Special Reference to its Carcinogenicity: An Interim Report, Rad. Prot. Dos. 16, 165-168. 